\title{
openheart Falling hospital and postdischarge mortality following CABG in New South Wales from 2000 to 2013
}

\author{
David B Brieger, ${ }^{\oplus}$ Austin C C Ng, ${ }^{2}$ Vincent Chow, ${ }^{3}$ Mario D'Souza, ${ }^{1}$ Karice Hyun, ${ }^{1}$ \\ Paul G Bannon, ${ }^{4}$ Leonard Kritharides ${ }^{1}$
}

\begin{abstract}
- Additional material is published online only. To view please visit the journal online (http://dx.doi.org/10.1136/ openhrt-2018-000959).
\end{abstract}

To cite: Brieger DB, Ng ACC, Chow V, et al. Falling hospital and postdischarge mortality following CABG in New South Wales from 2000 to 2013. Open Heart 2019;6:e000959.

doi:10.1136/

openhrt-2018-000959

Received 9 January 2019 Revised 24 January 2019 Accepted 10 February 2019

Check for updates

(c) Author(s) (or their employer(s)) 2019. Re-use permitted under CC BY-NC. No commercial re-use. See rights and permissions. Published by BMJ.

'Department of Cardiology, Concord Hospital, Sydney, New South Wales, Australia ${ }^{2}$ Department of Cardiology, Concord Hospital and The University of Sydney, Concord, New South Wales, Australia ${ }^{3}$ Department of Cardiology, Concord Hospital, The University of Sydney and ANZAC Medical

Research Institute, Sydney, New South Wales, Australia

${ }^{4}$ Cardiothoracic Surgery, Royal Prince Alfred Hospital, Camperdown, New South Wales, Australia

Correspondence to Dr David B Brieger; david. brieger@health.nsw.gov.au

\section{ABSTRACT}

Objectives To describe changes in mortality among patients undergoing coronary artery bypass grafting (CABG) in New South Wales (NSW) Australia from 2000 to 2013.

Methods Patients undergoing CABG were identified from the NSW Admission Patient Data Collection (APDC) registry, linked to the NSW state-wide death registry database. Changes in all-cause mortality over time were observed following stratification of the study cohort into two year groups.

Results We identified 54767 patients undergoing CABG during the study period. The risk profile of patients increased over time with significant increases in age, comorbidities and concomitant valve surgery (all $p<$ 0.0001 ). During a median follow-up period of 6 years, a total 12161 (22.2\%) of patients had died. Survival curves and adjusted analyses showed a steady fall in mortality rate: those operated on during 2012-2013 had $40 \%$ lower mortality than those operated on during 2000-2001 (HR $0.61 ; 95 \% \mathrm{Cl} 0.53$ to 0.69 ). This was contributed to both by a fall in mortality both in hospital ( $\mathrm{HR} 0.48,95 \% \mathrm{Cl}$ 0.37 to 0.62 ) and postdischarge (HR $0.73 ; 95 \% \mathrm{Cl} 0.61$ to 0.86).

Conclusions We report a consistent reduction in mediumterm mortality among a large unselected cohort of NSW patients undergoing CABG between 2000 and 2013. This fall is attributable both to an improvement in outcomes in hospital and in the postdischarge period.

\section{INTRODUCTION}

Coronary artery bypass grafting (CABG) is of proven prognostic benefit in patients with advanced coronary disease ${ }^{12}$ and remains one of the most commonly performed major surgical procedures. ${ }^{1}$ The risks are highest in the perioperative period and selecting a patient for surgical revascularisation involves weighing up the short-term risks against the accepted long-term benefit. ${ }^{1}$

Mortality among patients with coronary artery disease has fallen steadily since the $1960 \mathrm{~s} .{ }^{34}$ Thus, the decision regarding whether to subject patients to surgical revascularisation as opposed to medical therapy or percutaneous revascularisation would be better

\section{Key questions}

What is already known about this subject?

- Coronary artery bypass grafts are one of the most common major operations with significant morbidity and mortality.

What does this study add?

- In New South Wales, Australia, medium-term mortality has improved for these patients since 2000 reflecting improved survival both in hospital and following discharge.

How might this impact on clinical practice?

- Hospitals and clinicians should focus efforts on improvements in perioperative techniques, grafting strategies and long-term secondary prevention to ensure these improved outcomes are realised in all surgical centres.

informed by a contemporary understanding of both short-term risks of the procedure and long-term benefit. Several studies have shown an improvement in 30-day mortality in patients following CABG in the 10 years since $2000^{5}{ }^{6}$; however, while some reports have suggested an improvement in longer-term outcomes over time, ${ }^{7}$ others have not. ${ }^{6}$

The in-hospital mortality for CABG procedures in Australia was $2.7 \%$ in 2000, comparable to that reported internationally at that time, but greater than contemporary mortality rates. ${ }^{89}$ There are no longitudinal data examining whether the improvements demonstrated elsewhere have been replicated locally. Furthermore, there are few published data on longer-term outcomes following CABG in Australia.

The health administrative data collection system in New South Wales (NSW) houses information from all patients admitted to $>97 \%$ of public and private hospitals. This dataset contains information on patient comorbidities and all hospital procedures and is linked to the national death index. It therefore provides a valuable resource 
enabling the aggregation of large numbers of patient procedures and outcomes.

In this analysis, we have extracted information on CABG procedures performed in NSW from the years 2000 to 2013 with the goal of determining whether there had been any improvement in medium-term mortality over this time period. We also sought to evaluate the relative contribution of mortality during the in-hospital period and that in the postdischarge phase to any changes over time.

\section{METHODS}

\section{Study population}

Patients undergoing CABG between July 2000 and December 2013 were identified by Australian Classification of Health Interventions procedure codes from the NSW Admission Patient Data Collection (APDC) registry, held by the Centre for Health Record Linkage (CHeReL) database. Patients undergoing CABG were selected from a larger cohort of patients $(n=5$ 97805 admissions) undergoing a broader range of cardiac procedures including coronary angiography, percutaneous coronary intervention, electrophysiology procedures and transoesophageal echocardiography. Comparisons between this extracted dataset and a sample NSW health dataset of all patients with schaemic heart diseasei undergoing CABG from 2006 to 2011 (Better Cardiac Care Project) ${ }^{10}$ indicated that our study cohort represented approximately $90 \%$ of all CABG procedures in NSW.

\section{Patient variables}

The primary and secondary diagnoses associated with each CABG procedure were extracted from the APDC database. If the primary diagnosis of admission was listed as acute myocardial infarction (AMI), the CABG procedure was presumed to have occurred during the course of the admission precipitated by the acute coronary event. Performance of concomitant valve surgery (both repair and replacement) at the time of CABG was also collected.

\section{Study outcomes}

The primary outcome of the study was all-cause death at latest follow-up. Mortality was identified from the NSW state-wide death registry database also held by CHeReL, linked to the APDC registry. Cases were limited to only NSW state residents to minimise loss to follow-up through cross-border flows. In-hospital mortality and postdischarge mortality were also reported. Approval of the study protocol was granted by the NSW Population and Health Services Research Ethics Committee, reference number: 2013/09/479.

\section{Statistical analysis}

NSW residents admitted to hospital for CABG between 2 July 2000 and 28 December 2013 were included. All continuous variables are expressed as mean $\pm \mathrm{SD}$, unless otherwise stated, and categorical data given in frequency and percentages. For binary categorical variables, the Cochrane-Armitage trend test was used to determine whether there was a trend in the proportion across the periods. For the numeric variables, linear regression analysis was used with the year group variable treated as a continuous variable. The $p$ value was used to determine whether there was a linear trend in the means.

Kaplan-Meier methods were used to determine survival rates. This was limited to the cohort who underwent surgery before July 2013 to ensure 6-month follow-up for all. To compare the changes in CABG mortality over time, the study cohort was stratified into seven independent time groups 2000-2001, 2002-2003, 2004-2005, 2006-2007, 2008-2009, 2010-2011 and 2012-2013. Survival curves were constructed to allow comparisons between those seven groups. Changes in total mortality over those seven groups were compared after adjustment for confounding effects, using Cox regression analyses. To ascertain the relative contribution of in-hospital and postdischarge factors to changes in the outcome, in-hospital mortality was determined, both before and after adjustment using Cox regression analyses. Factors used for adjustment for these analyses included age, gender, comorbidities including prior percutaneous coronary interventions, coronary artery bypass graft, congestive cardiac failure, atrial fibrillation stroke, peripheral vascular disease, diabetes, current/ ex-smoker, chronic pulmonary disease and chronic kidney disease, indication for procedure (AMI vs not) and performance of valve surgery at the time of CABG.

The robustness of the findings was confirmed in additional analyses restricted to patients with complete follow-up times. Mortality was determined at 6 months (including the cohort to July 2013), 2 years (restricted to the populations with complete ascertainment from 2000 to 2011) and 5 years (restricted to those with complete ascertainment from 2000 to 2007). In an additional sensitivity analysis, long-term mortality was evaluated following restriction of the population to those surviving 30 days postdischarge to be consistent with many post-CABG survival models and quality metrics. ${ }^{11}$

All analyses were performed using SAS V.9.3 (IBM, USA). A two-tailed probability value $<0.05$ was considered statistically significant. All authors had full access to all the data in the study, and the corresponding author had final responsibility for the decision to submit for publication.

\section{RESULTS \\ Description of study population}

Our initial data extract included almost $600 \quad 000$ patient records. From these, 54767 patients undergoing in-hospital CABG for whom the dates of surgery were available comprised the study cohort (figure 1). The risk profile of the patient population steadily 

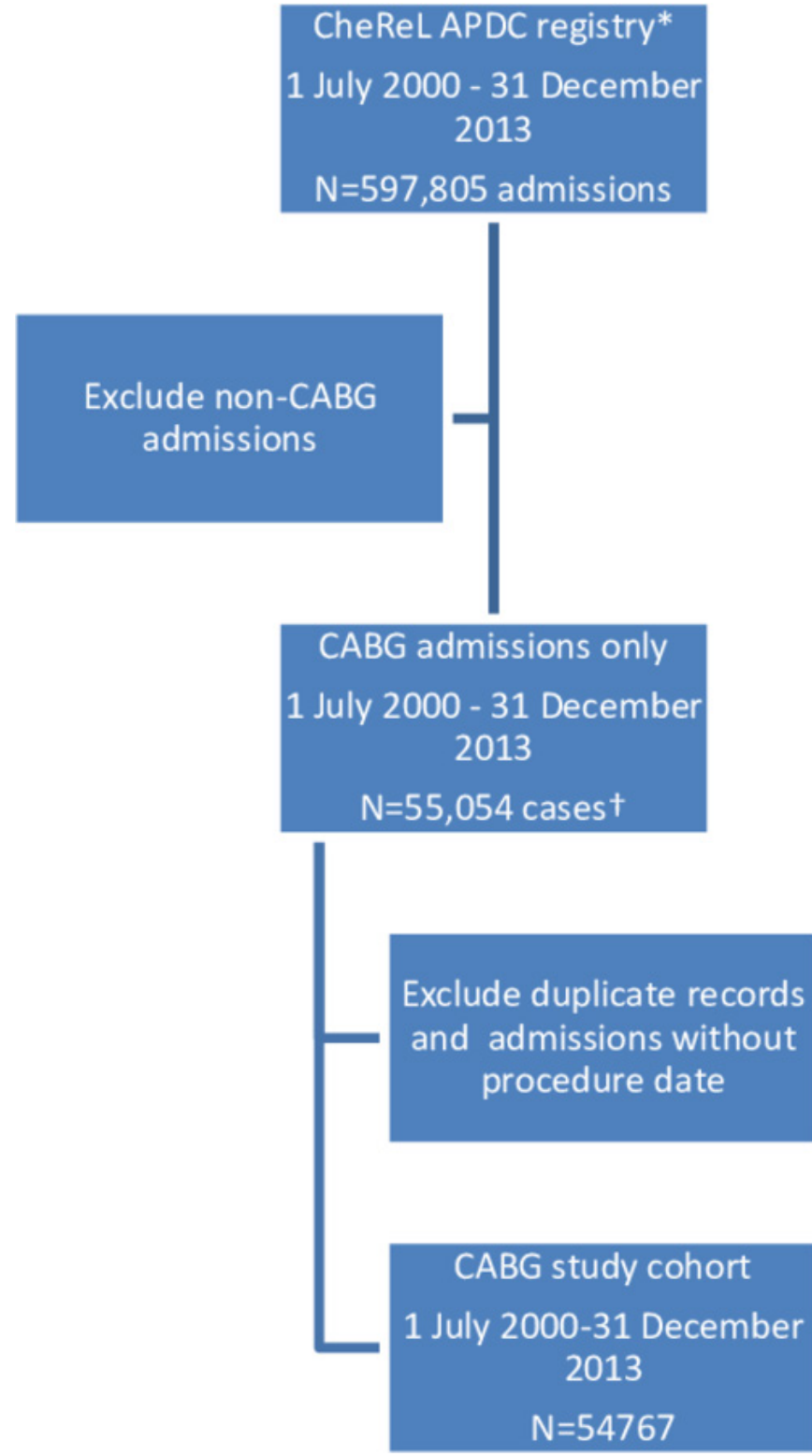

Figure 1 Derivation of CABG cohort. APDC, Admission Patient Data Collection; CABG, coronary artery bypass grafting; CheRel, Centre for Health Record Linkage.

increased over time, with significant increases noted in age, proportion of males, cardiac comorbidities, diabetes and chronic kidney disease (all $\mathrm{p}<0.0001)$. A primary admission diagnosis of AMI among patients undergoing CABG increased (14\%-27\%, p<0.0001) during the study period. Concomitant valve surgery at the time of CABG more than doubled over the time period $(9 \%-19 \%, \mathrm{p}<0.0001)$ (table 1$)$.

\section{Overall mortality during the study period}

During a median follow-up period of 6 years, a total $12161(22.2 \%)$ of patients had died. Survival curves for each cohort showed a steady fall in mortality rate (figure 2).

Following adjustment, this trend was more apparent. Mortality at follow-up among those operated on during
2006-2007 was 11\% lower (HR 0.89; 95\% CI 0.83-0.95) and those having CABG in 2012-2013 were $40 \%$ lower (HR $0.61 ; 95 \%$ CI0.53-0.69) than those operated on during 2000-2001 (figure 3A).

\section{In-hospital mortality}

A total of 1337 (2.4\%) of patients died during their hospital admission. There was a steady fall in in-hospital mortality from 2004 to 2005 (2.9\%) to 2012-2013 (1.6\%), $\mathrm{p}<0.0001$ (table 2). When compared with that observed in 2000-2001, the adjusted HR of death had fallen by $30 \%$ by $2008-2009$ (HR $0.71,95 \%$ CI 0.57 to 0.89 ), and more than $50 \%$ by $2012-2013$ (HR $0.48,95 \%$ CI 0.37 to 0.62 ) (figure $3 \mathrm{~B}$ ).

\section{Postdischarge mortality}

An adjusted landmark long-term survival analysis was performed restricted to patients surviving to hospital discharge. Compared with patients operated on during 2000-2001, the mortality hazard was more than $25 \%$ less among patients undergoing CABG from 2012 to 2013 (HR $0.73 ; 95 \%$ CI 0.61 to 0.86 ) (figure 3C) indicating that postdischarge factors contributed to the improved outcomes in this patient cohort.

\section{Sensitivity analyses}

A further landmark analysis was performed limited to patients who survived to 30 days postdischarge. Similar results were obtained to those observed among postdischarge survivors (2012/2003 vs 2000/2001 adjusted HR 0.79, 95\%; CI 0.65 to 0.94) (online supplementary appendix figure 1).

To further control for differing follow-up durations among patients having their surgery performed at different times, a series of analyses were performed limited to patient cohorts for whom there was complete follow-up. Mortality at 6 months was consistent with the longer-term result: (2012/2003 vs 2000/2001 adjusted HR 0.64; $95 \%$ CI 0.53 to 0.78 ) (online supplementary appendix figure 2). Two-year follow-up data were available for patients operated on up to 2012 and again a benefit was apparent for this cohort relative to those operated on earlier in the study period (2011/2002 vs 2000/2001 adjusted HR 0.76; 95\% 0.67-0.88) (online supplementary appendix figure $3)$. Complete 5-year follow-up was limited to the cohort of patients undergoing surgery earlier during the study period (latest 2007) and the benefit in mortality was attenuated in this cohort when compared with those undergoing CABG earlier:2000/2001 vs 2006/2007 (adjusted HR 0.94; 95\% CI 0.86 to 1.03 ) (online supplementary appendix figure 4 ).

\section{DISCUSSION}

This large dataset of the majority patients undergoing CABG in NSW hospitals from 2000 to 2013 shows a $40 \%$ decline in medium-term mortality during this time period. This has occurred despite an increase in the clinical risk profile of this cohort. In-hospital mortality fell from $2.5 \%$ to $1.6 \%$, and after adjusting for patient risk, the hazard of dying in 
Table 1 Demographics and background history of patients undergoing CABG in New South Wales from 2000 to 2013

\begin{tabular}{|c|c|c|c|c|c|c|c|c|c|}
\hline Variable & $\begin{array}{l}2000- \\
2001 \\
\text { n (\%) } \\
N=7318\end{array}$ & $\begin{array}{l}2002- \\
2003 \\
\text { n (\%) } \\
N=9563\end{array}$ & $\begin{array}{l}2004- \\
2005 \\
n(\%) \\
N=8420\end{array}$ & $\begin{array}{l}2006- \\
2007 \\
\text { n (\%) } \\
N=8036\end{array}$ & $\begin{array}{l}2008- \\
2009 \\
\text { n (\%) } \\
N=7452\end{array}$ & $\begin{array}{l}2010- \\
2011 \\
n(\%) \\
N=6782\end{array}$ & $\begin{array}{l}2012- \\
2013 \\
n(\%) \\
N=7196\end{array}$ & $\begin{array}{l}\text { Overall } \\
n(\%) \\
N=54767\end{array}$ & $P$ value \\
\hline Male & $5524(75)$ & $7211(75)$ & $6474(77)$ & $6230(78)$ & $5815(78)$ & $5363(79)$ & $5768(80)$ & $42385(77)$ & $<0.0001$ \\
\hline Age, mean (SD) & $66.5(10.6)$ & $67.0(10.5)$ & $67.5(10.6)$ & $67.8(10.7)$ & $68.0(10.7)$ & $67.9(10.6)$ & $67.8(10.6)$ & $67.5(10.6)$ & $<0.0001$ \\
\hline $\begin{array}{l}\text { Acute myocardial infarct } \\
\text { indication for } C A B G\end{array}$ & $1018(14)$ & $1608(17)$ & $1826(22)$ & $1996(25)$ & $1951(26)$ & $1852(27)$ & $1933(27)$ & $12184(22)$ & $<0.0001$ \\
\hline Atrial fibrillation \& Flutter & $1835(25)$ & $2793(29)$ & $2861(34)$ & $2853(36)$ & $2814(38)$ & 2642 (39) & $2809(39)$ & $18607(34)$ & $<0.0001$ \\
\hline Congestive heart failure & $519(7)$ & $718(8)$ & $817(10)$ & $728(9)$ & $693(9)$ & $642(9)$ & $690(10)$ & 4807 (9) & $<0.0001$ \\
\hline Cerebrovascular accident & $116(2)$ & $161(2)$ & $157(2)$ & $140(2)$ & $110(1)$ & $112(2)$ & $98(1)$ & $894(2)$ & 0.1376 \\
\hline Chronic kidney disease & $361(5)$ & $547(6)$ & $643(8)$ & $738(9)$ & $678(9)$ & $628(9)$ & $715(10)$ & $4310(8)$ & $<0.0001$ \\
\hline Chronic pulmonary disease & $581(8)$ & $664(7)$ & $705(8)$ & $531(7)$ & $323(4)$ & $273(4)$ & $312(4)$ & $3389(6)$ & $<0.0001$ \\
\hline Current and past tobacco use & $3418(47)$ & $4830(51)$ & $4345(52)$ & $4421(55)$ & $4046(54)$ & $3754(55)$ & $4010(56)$ & $28824(53)$ & $<0.0001$ \\
\hline Current tobacco use & $780(11)$ & $992(10)$ & $1005(12)$ & $952(12)$ & $931(12)$ & $837(12)$ & $951(13)$ & $6448(12)$ & $<0.0001$ \\
\hline Diabetes & $1592(22)$ & $2544(27)$ & $2410(29)$ & $2591(32)$ & $2060(28)$ & $1302(19)$ & $2290(32)$ & $14789(27)$ & $<0.0001$ \\
\hline Hypertension & $4311(59)$ & $5981(63)$ & $5667(67)$ & $5606(70)$ & $4941(66)$ & $4322(64)$ & $4872(68)$ & $35700(65)$ & $<0.0001$ \\
\hline Peripheral vascular disease & $563(8)$ & $824(9)$ & $804(10)$ & $688(9)$ & $530(7)$ & $408(6)$ & $462(6)$ & $4279(8)$ & $<0.0001$ \\
\hline Prior $\mathrm{CABG}$ & $267(4)$ & $387(4)$ & $262(3)$ & $214(3)$ & $160(2)$ & $143(2)$ & $120(2)$ & $1553(3)$ & $<0.0001$ \\
\hline $\begin{array}{l}\text { Prior percutaneous coronary } \\
\text { intervention }\end{array}$ & $461(6)$ & $578(6)$ & $561(7)$ & $633(8)$ & $565(8)$ & $563(8)$ & $619(9)$ & $3980(7)$ & $<0.0001$ \\
\hline $\begin{array}{l}\text { Valve surgery at the time of } \\
\text { CABG }\end{array}$ & $682(9)$ & $1021(11)$ & $1148(14)$ & $1232(15)$ & 1294 (17) & 1219 (18) & 1345 (19) & 7941 (14) & $<0.0001$ \\
\hline
\end{tabular}

CABG, coronary artery bypass grafting.

hospital fell by $50 \%$. There were additional mortality gains post-hospital discharge with a $27 \%$ reduced hazard from 2000-2001 to 2012-2013, a finding which was only slightly attenuated (21\% reduced hazard) when medium- term survival was evaluated among patients surviving 30 days following their operation.

Australian studies examining temporal trends in outcomes following CABG have reported conflicting data

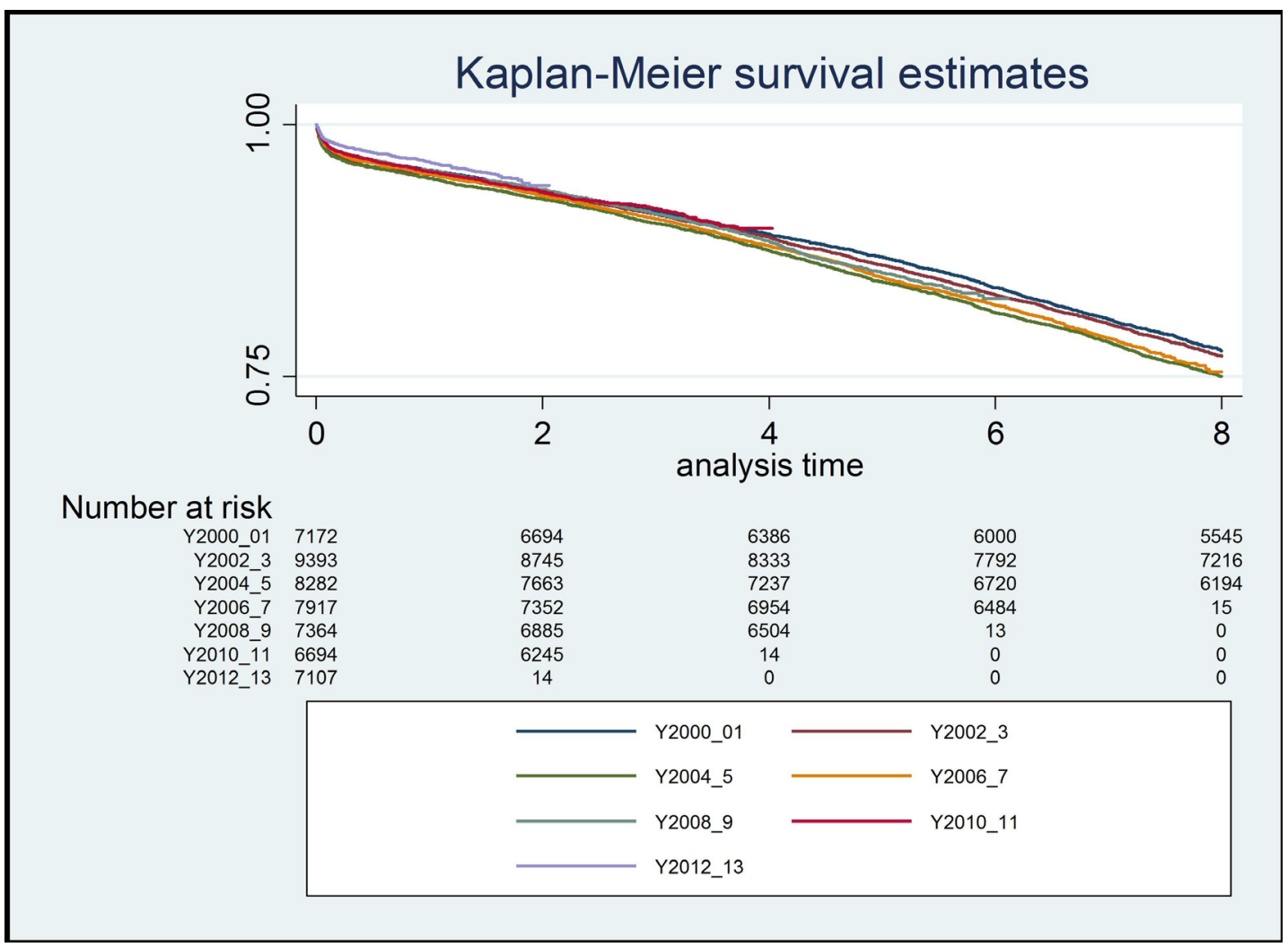

Figure 2 Kaplan-Meier survival estimates for the whole study cohort. 
A

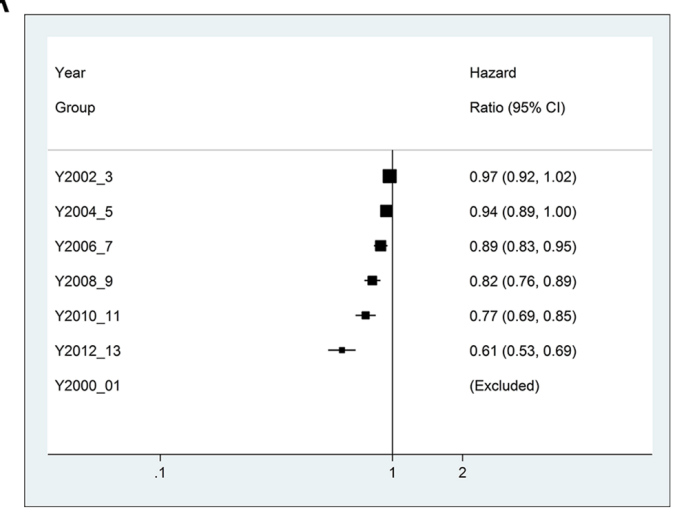

B

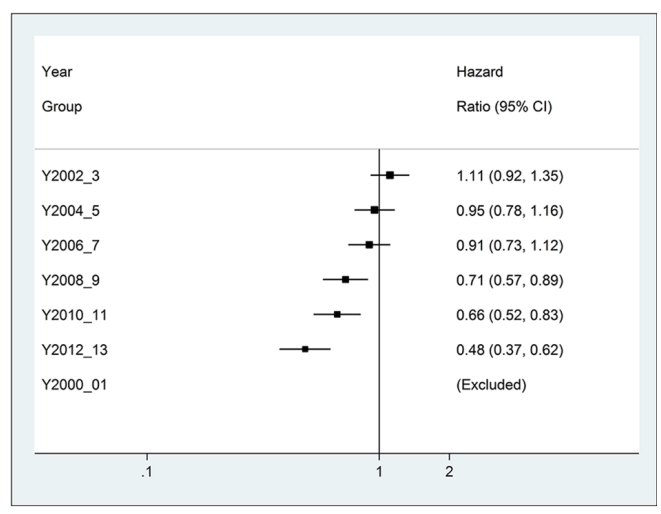

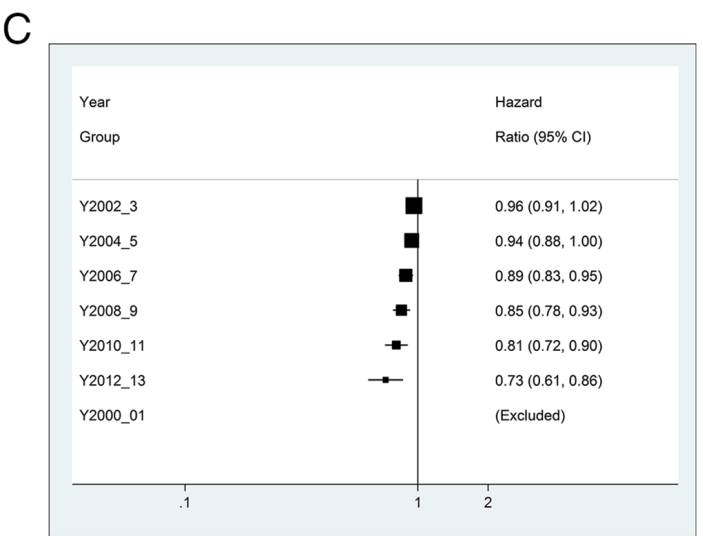

Figure 3 (A) Forest plot showing adjusted long-term mortality by year groupings. (B) Forest plot showing adjusted in hospital mortality by year groupings. (C) Forest plot showing adjusted postdischarge mortality by year groupings.

on short-term outcomes. An early report of 8910 patients from a Western Australian cardiothoracic surgical database reported worsening 30-day mortality from 1980 to 1993 attributable to inclusion of higher risk patients. ${ }^{12}$ However, a more recent report from the Australian and New Zealand Society of Cardiac and Thoracic Surgeons database including 27 hospitals nationally reported a decline in 30-day mortality from 2009 to $2013 .{ }^{13}$ These findings are consistent with ours; it was in later years in our study that the improvement in hospital outcomes became apparent. Our data are also comparable to those from a recent Danish study which reported a reduction in 30-day mortality from $4.1 \%$ 1999-2000 to 2.4\% 2011-2012, ${ }^{6}$ and a report from the US Society of Thoracic Surgeons database which reported a fall in 30-day mortality from $2.4 \%$ in 2000 to $1.9 \%$ in $2009 .^{5}$

There have been a number of developments in anaesthetic and surgical technique that may have contributed to the improvement in perioperative outcomes. These include bleeding and transfusion avoidance strategies, accelerated extubation and optimising perioperative analgesia. ${ }^{14}$ The performance of procedures without cardiopulmonary bypass (off pump) may improve short- term outcomes in high-risk patients, although there is no evidence that the uptake of this procedure has increased over this time period. ${ }^{91315}$

Studies that have investigated changes in longer-term mortality have also yielded conflicting results. ${ }^{67} \mathrm{~A}$ Danish cohort study found no improvement in 1-year mortality from 1999 to 2012 which they attributed to the increasing age of their cohort despite adjustment. This study was limited to only three contributing hospitals, which might explain the disparate findings. ${ }^{6}$ In contrast, a population-based study from Sweden reported an improvement in 4-year survival among most CABG patients from 1987 to $2006 .{ }^{7}$ The finding of a decline in long-term mortality is plausible and may be attributable to a combination of better grafting strategies and better secondary prevention. Improved vein graft handling has been shown to improve graft patency. ${ }^{16}$ All arterial grafting has been associated

\begin{tabular}{|c|c|c|c|c|c|c|c|c|c|}
\hline Variable & $\begin{array}{l}2000- \\
2001 \\
n(\%) \\
N=7318\end{array}$ & $\begin{array}{l}2002- \\
2003 \\
n(\%) \\
N=9563\end{array}$ & $\begin{array}{l}2004- \\
2005 \\
n(\%) \\
N=8420\end{array}$ & $\begin{array}{l}2006- \\
2007 \\
\text { n (\%) } \\
N=8036\end{array}$ & $\begin{array}{l}2008- \\
2009 \\
\text { n (\%) } \\
\text { N=7452 }\end{array}$ & $\begin{array}{l}2010- \\
2011 \\
n(\%) \\
N=6782\end{array}$ & $\begin{array}{l}2012- \\
2013 \\
n(\%) \\
N=7196\end{array}$ & $\begin{array}{l}\text { Overall } \\
n(\%) \\
N=54767\end{array}$ & $P$ value \\
\hline In-hospital death & $186(2.5)$ & $263(2.8)$ & $247(2.9)$ & $214(2.7)$ & $166(2.2)$ & $143(2.1)$ & $118(1.6)$ & $1337(2.4)$ & $<0.0001$ \\
\hline
\end{tabular}


with improved outcomes in some series ${ }^{18}$ and is increasing in selected hospitals around Australia. ${ }^{1319}$ Continued statin and antiplatelet therapies improve longer-term outcomes following revascularisation. ${ }^{20}$ Increased uptake of these medications has been documented to have occurred over this period in Australian studies. ${ }^{21}$

In additional analyses restricted to patients in whom there was complete follow-up, the improvement in mortality was attenuated in the years following CABG. Patients undergoing surgery in 2000-2001 had a 25\% greater likelihood of death by 2 years than those having surgery in 2012-2013; in contrast, there was no significant improvement in 5-year survival among the patients for whom there was complete follow-up. There are several explanations for this. First, these patients underwent surgery earlier in the study period, at a time when relative improvements in mortality in our cohort were less apparent. Second, the benefits of bypass surgery relative to medical therapy attenuate over time. ${ }^{222} 23$ This is thought to be primarily attributable to bypass graft attrition $^{24} 25$ and may also be contributed to by competing risk from other causes of death in older populations. ${ }^{23}$ As discussed above, improved vein preservation techniques and increasing use of arterial conduits may improve longterm graft patency and survival. ${ }^{9} 1626$

Several limitations of our study deserve mention. First, the data were derived from administrative and procedural codes which are subject to error ${ }^{27}{ }^{28}$ In addition, we were limited by a lack of procedural details, such as vein graft preservation techniques used, relative changes in the prevalence of arterial grafting and perioperative medications. This meant we were unable to document changes in practice that may have contributed to the observed improvement in perioperative outcomes. Furthermore, we did not have data on long-term medication use or attendance at cardiac rehabilitation in our cohort. This information would allow a determination of the contribution of these factors to improving outcomes. As this cohort was extracted from a broader group of patients undergoing a range of cardiac procedures, it includes only about $90 \%$ of CABG procedures performed over this time period. However, we do not believe the method of identification introduces any bias to the population and so should not influence our findings.

In conclusion, we report a consistent reduction in medium-term mortality among a large unselected cohort of NSW patients undergoing CABG between 2000 and 2013. This fall is attributable both to an improvement in outcomes in hospital and in the postdischarge period. The age-standardised death rate for coronary heart disease in Australia has fallen by $4.5 \%$ per year between 1980 and 2015. This has been attributed to improvements in disease prevention through risk factor control, better emergency medical care for heart attack and an increase in specialised coronary care units. ${ }^{29}$ In this paper, we identify an additional important contemporary contributor to this improvement in mortality, better survival among patients undergoing CABG.
Contributors Planning of the study: DB, AN and MD. Performance of the analyses: $\mathrm{MD}$ and $\mathrm{KH}$. Reporting of the work: DB, AN, VC, MD, KH, PB and LK. Responsible for overall content: DB.

Funding Funding for data linkage was provided by the Department of Cardiology, Concord hospital.

Competing interests None declared.

Patient consent for publication Not required.

Provenance and peer review Not commissioned; externally peer reviewed.

Open access This is an open access article distributed in accordance with the Creative Commons Attribution Non Commercial (CC BY-NC 4.0) license, which permits others to distribute, remix, adapt, build upon this work non-commercially, and license their derivative works on different terms, provided the original work is properly cited, appropriate credit is given, any changes made indicated, and the use is non-commercial. See: http://creativecommons.org/licenses/by-nc/4.0/.

\section{REFERENCES}

1. Alexander $\mathrm{JH}, \mathrm{Smith} P K$. Coronary-artery bypass grafting. $N$ Engl $J$ Med 2016;374:1954-64.

2. Yusuf S, Zucker D, Peduzzi P, et al. Effect of coronary artery bypass graft surgery on survival: overview of 10 -year results from randomised trials by the coronary artery bypass graft surgery Trialists collaboration. Lancet 1994;344:563-70.

3. Ford ES, Capewell S. Proportion of the decline in cardiovascular mortality disease due to prevention versus treatment: public health versus clinical care. Annu Rev Public Health 2011;32:5-22.

4. Hall M, Dondo TB, Yan AT, et al. Association of clinical factors and therapeutic strategies with improvements in survival following non-ST-elevation myocardial infarction, 2003-2013. JAMA 2016;316:1073-82.

5. ElBardissi AW, Aranki SF, Sheng S, et al. Trends in isolated coronary artery bypass grafting: an analysis of the Society of thoracic surgeons adult cardiac surgery database. J Thorac Cardiovasc Surg 2012;143:273-81.

6. Hansen LS, Hjortdal VE, Andreasen JJ, et al. 30-day mortality after coronary artery bypass grafting and valve surgery has greatly improved over the last decade, but the 1-year mortality remains constant. Ann Card Anaesth 2015;18:138-42.

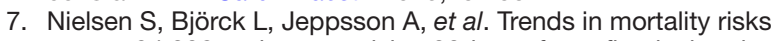
among 94,328 patients surviving 30 days after a first isolated coronary artery bypass graft procedure from 1987 to 2006: a population-based study. Int J Cardiol 2017;244:316-21.

8. Australian Institute of Health and Welfare. Coronary revascularisation in Australia 2000. Canberra: AlHW, 2003.

9. Head SJ, Milojevic M, Taggart DP, et al. Current practice of state-of-the-art surgical coronary revascularization. Circulation 2017;136:1331-45.

10. NSW Health. Better cardiac care. Available: http://www.health.nsw. gov.au/aboriginal/bettercardiaccare/Pages/activities.aspx [Accessed Jul 2018].

11. Shahian DM, O'Brien SM, Sheng S, et al. Predictors of long-term survival after coronary artery bypass grafting surgery: results from the Society of thoracic surgeons adult cardiac surgery database (the ASCERT study). Circulation 2012;125:1491-500.

12. Bradshaw PJ, Jamrozik K, Le M, et al. Mortality and recurrent cardiac events after coronary artery bypass graft: long term outcomes in a population study. Heart 2002;88:488-94.

13. Tran L, Marrow N, Ayres C, et al. The Australian and New Zealand Society of cardiac and Thoracic surgeons (ANZSCTS) national cardiac database program national annual report 2014, 2015. Available: http://anzscts.org/wp-content/uploads/2016/12/ ANZSCTS-National-Annual-Report-2014.pdf [Accessed Aug 2018]

14. Hillis LD, Smith PK, Anderson JL, et al. 2011 ACCF/AHA guideline for coronary artery bypass graft surgery. A report of the American College of cardiology Foundation/American Heart Association Task Force on practice guidelines. developed in collaboration with the American Association for thoracic surgery, society of cardiovascular Anesthesiologists, and Society of thoracic surgeons. J Am Coll Cardiol 2011;58:e123-210.

15. Kowalewski M, Pawliszak W, Malvindi PG, et al. Off-pump coronary artery bypass grafting improves short-term outcomes in high-risk patients compared with on-pump coronary artery bypass grafting: meta-analysis. J Thorac Cardiovasc Surg 2016;151:60-77.

16. Samano N, Geijer H, Liden M, et al. The no-touch saphenous vein for coronary artery bypass grafting maintains a patency, after 16 years, comparable to the left internal thoracic artery: a randomized trial. $J$ Thorac Cardiovasc Surg 2015;150:880-8. 
17. Harskamp RE, Alexander JH, Schulte PJ, et al. Vein graft preservation solutions, patency, and outcomes after coronary artery bypass graft surgery: follow-up from the prevent IV randomized clinical trial. JAMA Surg 2014;149:798-805.

18. Gaudino M, Puskas JD, Di Franco A, et al. Three arterial grafts improve late survival: a meta-analysis of Propensity-Matched studies. Circulation 2017;135:1036-44.

19. Tran L, Dahya D, Huq M. ANZSCTS cardiac surgery database program. Annual report 2011, 2012. Available: http://anzscts.org/wpcontent/uploads/2016/12/ANZSCTS-National-Annual-Report-2011. pdf [Accessed Aug 2018].

20. Iqbal J, Zhang Y-J, Holmes DR, et al. Optimal medical therapy improves clinical outcomes in patients undergoing revascularization with percutaneous coronary intervention or coronary artery bypass grafting: insights from the synergy between percutaneous coronary intervention with Taxus and cardiac surgery (SYNTAX) trial at the 5-year follow-up. Circulation 2015;131:1269-77.

21. Chan T, Dabin B, Hyun K, et al. Falling cholesterol trend at acute coronary syndrome presentation is strongly related to statin use for secondary prevention. Int J Cardiol 2016;212:192-7.

22. Varnauskas E. Twelve-year follow-up of survival in the randomized European coronary surgery study. N Engl J Med 1988;319:332-7.
23. van Domburg RT, Kappetein AP, Bogers AJJC. The clinical outcome after coronary bypass surgery: a 30-year follow-up study. Eur Heart $J$ 2009;30:453-8.

24. Bourassa MG, Fisher LD, Campeau L, et al. Long-term fate of bypass grafts: the coronary artery Surgery Study (CASS) and Montreal heart Institute experiences. Circulation 1985;72.

25. Goldman S, Zadina K, Moritz T, et al. Long-term patency of saphenous vein and left internal mammary artery grafts after coronary artery bypass surgery: results from a department of Veterans Affairs Cooperative study. J Am Coll Cardiol 2004;44:2149-56.

26. Yanagawa B, Verma S, Mazine A, et al. Impact of total arterial revascularization on long term survival: a systematic review and meta-analysis of 130,305 patients. Int J Cardiol 2017;233:29-36.

27. Kelman CW, Bass AJ, Holman CDJ. Research use of linked health data--a best practice protocol. Aust N Z J Public Health 2002;26:251-5.

28. Henderson T, Shepheard J, Sundararajan V. Quality of diagnosis and procedure coding in ICD-10 administrative data. Med Care 2006;44:1011-9.

29. Australian Institute of Health and Welfare. Trends in cardiovascular deaths. Canberra: AlHW, 2017. 